\title{
Magnetic energy-barrier distributions for ferrihydrite nanoparticles formed by reconstituting ferritin
}

\author{
N.T. Gorham ${ }^{1,2}$, T.G. St Pierre ${ }^{1}$, W. Chua-anusorn ${ }^{1}$, and G.M. Parkinson ${ }^{2}$ \\ ${ }^{1}$ School of Physics, M013, The University of Western Australia, Crawley, WA 6009, \\ Australia \\ ${ }^{2}$ Nanochemistry Research Institute, Curtin University of Technology, Bentley, WA 6102, \\ Australia
}

To be submitted to Journal of Applied Physics

Keywords: ferritin; ferrihydrite; surface effects; nanoparticles; magnetic relaxation 


\begin{abstract}
The spherical cage-like protein ferritin was reconstituted with varying numbers of iron atoms per protein shell (ranging from approximately 20 to 1100) at temperatures of both 25 and $50^{\circ} \mathrm{C}$ to produce iron(III) oxyhydroxide (ferrihydrite) particles with different average particle sizes and degrees of crystallinity. After characterisation of the structural properties of the resulting iron-oxyhydroxide nanoparticles with transmission electron microscopy and Mössbauer spectroscopy, magnetic viscosity measurements were made in zero applied magnetic field and the resulting data were used to calculate the apparent magnetic-moment-weighted energy barrier distributions for the samples. The distributions measured were typically comprised of both a lognormal distribution and an exponential decay of barrier frequency with increasing barrier height. Evidence that the lognormal component of this distribution arises from the distribution of particle volumes and moments within the ensemble is strongly supported by the increase in the mode of the energy barrier distribution with increasing particle size. The exponentially decaying distribution has a relatively higher contribution to the overall distribution for the more crystalline reconstituted ferritin samples suggesting that it may be associated with uncompensated spins at particle surfaces.
\end{abstract}




\section{Introduction}

Since the pioneering work of Stoner and Wohlfarth ${ }^{1}$ and Néel ${ }^{2}$ nearly six decades ago, the magnetism of single-domain particles has been an active area of research. In the field of nanomagnetism, fundamental magnetic relaxation properties such as quantum tunnelling of magnetisation (where a macroscopic ensemble of atomic magnetic moments collectively tunnels through an effective energy barrier much larger than the thermal energy) have been investigated in extensive detail by many authors (see, e.g., ${ }^{3-7}$ ). Another related area of interest is surface related effects (see, e.g., ${ }^{8-10}$ ), where the increasing role of the surface spins as particle size is reduced results in substantially different magnetic properties from those observed in their bulk counterparts.

Experimental studies of fundamental issues are nearly always complicated by the variety of parameters that govern the overall physical behaviour of a system. One very significant parameter influencing structural and dynamic properties of real systems of magnetic nanoparticles is the interaction between the particles. For example, it has been observed that, owing to dipolar interactions, nanoparticle systems may completely change their magnetic behaviour from superparamagnetic to spin glass like ${ }^{11}$. Whilst such results are themselves of interest, the focus here will be on the study of antiferromagnetic particles encapsulated in protein cages (ferritin). A ferritin protein molecule is approximately spherical with an external diameter of approximately $12 \mathrm{~nm}$ and an internal cavity with diameter approximately $8 \mathrm{~nm}$. Iron(III) oxyhydroxide particles can be formed within the cavities via precipitation from solution. Such systems have very weak magnetic dipole moments so that dipole-dipole interactions are negligible. The 
organic matrix surrounding the iron(III) oxyhydroxide particles ensures sufficient separation between particles to prevent interparticle exchange coupling. These two characteristics enable the intrinsic properties of the particles to be directly assessed without the complication of interparticle magnetic interactions. Here, we report data on the magnetic viscosity of ferritin that has been reconstituted with iron(III) oxyhydroxide to obtain nanoparticles with different average particle size and crystallinity. The data shed further light on the magnetic relaxation processes of magnetic nanoparticles at temperatures above $1 \mathrm{~K}$.

\section{Materials and methods}

Horse spleen apoferritin in $0.1 \mathrm{M}$ sodium acetate buffer $(\mathrm{pH}$ 5.3) was obtained from Sigma Chemical Company. Dialysis techniques were utilised to remove any excess metal atoms in the solution. The concentration of apoferritin was firstly determined by using UV/visible spectrophotometry at $280 \mathrm{~nm}$ following methods described elsewhere ${ }^{12}$. A Hewlett Packard 8450A UV/VIS spectrophotometer was used for these measurements. Apoferritin $(1 \mathrm{mg} / \mathrm{ml})$ in MOPS buffer $(0.1 \mathrm{M}, \mathrm{pH} 7.5)$ was reconstituted by additions of $10 \mathrm{mM} \mathrm{FeSO}_{4}$ solution to give theoretical loadings of 50, 100, 500, 1000, 1500 and 2000 iron atoms per ferritin molecule at 25 and $50^{\circ} \mathrm{C}$. It has been shown previously that the temperature of the solution during the precipitation process has an effect on the structure of the resulting mineral cores with higher temperatures yielding more crystalline particles 13,14. The reaction was carried out in a stepwise fashion by loading 50 iron atoms per molecule of protein every hour, with both solutions kept at a constant temperature in a 
water-bath. After filtering, drops of the reconstituted ferritin solutions were prepared for transmission electron microscopy study with the remainder being freeze-dried. The morphology and size of the mineral cores of the reconstituted ferritins were studied using a transmission electron microscope (TEM) operated at $80 \mathrm{keV}$. Drops of each of the ferritin samples diluted to about $0.5 \mathrm{mg} / \mathrm{ml}$ were allowed to dry onto formvar coated electron microscope copper grids which were subsequently air-dried. The particle size distribution of the ferritin samples was determined by using Vernier callipers to measure the lengths of the longest axis on enlarged copies of the micrographs. A minimum of 146 particles was measured for each sample. Catalase crystals (Alltech $\left.{ }^{\circledR}\right)$, which have lattice spacings of $8.75 \mathrm{~nm}$ and $6.85 \mathrm{~nm}$, were used to calibrate the magnification of the TEM.

Each freeze-dried reconstituted ferritin sample was mounted in $10 \mathrm{~mm}$ diameter perspex sample holders for ${ }^{57} \mathrm{Fe}$ Mössbauer spectroscopy measurements. The methods used and the results obtained from Mössbauer spectroscopy on these samples are described elsewhere ${ }^{14}$.

All magnetic measurements were performed using a commercial SQUID-based magnetometer (Quantum Design MPMS-7). Zero-field cooled and 70-kOe field cooled hysteresis loops were measured at temperatures of 5, 7, 9 and $11 \mathrm{~K}$ on all the reconstituted ferritins. A field spacing of approximately $1 \mathrm{kOe}$ was maintained for all hysteresis measurements. Low temperature magnetic time dependent measurements were performed on all samples at temperatures ranging from 2 to $22 \mathrm{~K}$. Each viscosity measurement involved zero-field cooling the sample from the superparamagnetic state down to the target temperature, applying and subsequently removing a large applied field 
$\left(\mathrm{H}_{\mathrm{tmp}}=70 \mathrm{kOe}\right)$ and then recording the thermoremanent magnetisation as a function of time for approximately $10^{3} \mathrm{~s}$.

\section{Experimental results}

\subsection{Sample characterisation}

The experimental results of the sample characterisation are summarised here and shown in detail in ${ }^{14}$.

On addition of the $\mathrm{Fe}(\mathrm{II})$ solutions to the apoferritin solutions no visible precipitation was observed to occur for loadings of 1500 iron atoms per ferritin shell or less at $25^{\circ} \mathrm{C}$ and for loadings of $1000 \mathrm{Fe}$ atoms per shell or less at $50^{\circ} \mathrm{C}$. At 2000 iron atoms per shell at $25^{\circ} \mathrm{C}$ and 1500 iron atoms per shell at $50^{\circ} \mathrm{C}$, there was evidence for a small amount of precipitation in the resulting solutions (i.e. precipitation occurring outside of the protein shells). For the samples in which no visible precipitation was observed, a good linear correlation was found between the nominal number of iron atoms added per protein shell and the number of iron atoms per protein shell deduced from elemental analysis of the iron content of the freeze dried reconstituted ferritin. For the two samples that showed a small amount of visible precipitation during preparation, the ratios of numbers of iron atoms per shell measured for the freeze dried material to the nominal number of iron atoms per shell were found to be slightly lower than for the samples with no precipitation.

The particle size distributions measured from the transmission electron micrographs of the ferritins reconstituted at 25 and $50^{\circ} \mathrm{C}$ (and shown in Table I) indicate maximum particle sizes of approximately $8 \mathrm{~nm}$. This observed maximum limit for the particle size 
is consistent with the known dimensions of horse spleen apoferritin. The relationship between the mean particle volume measured by TEM and the measured iron to protein ratio suggests a density of $6.4 \pm 0.6$ iron atoms per $\mathrm{nm}^{3}$ for the mineral particles ${ }^{14}$.

Mössbauer spectroscopy indicates that all particles have a high degree of structural

disorder ${ }^{14}$. Particles precipitated at the higher temperature tend to have slightly higher degrees of structural order compared with their counterparts precipitated at the lower temperature ${ }^{14}$.

\subsection{Magnetometry results}

For temperatures below $11 \mathrm{~K}$ the magnetisation versus applied field curves measured for all reconstituted ferritins remain open near the origin and display a non-zero remanence and coercivity. In addition, when measured after cooling in an applied field, each hysteresis loop exhibits a shift along the field axis which rapidly decays to zero with increasing temperature of measurement. The data are qualitatively similar to those measured previously for native ferritin at $5 \mathrm{~K}^{15}$.

Figure 1 shows the decay with time of the remanent iron specific magnetisation of two of the reconstituted ferritin samples after removal of a 70-kOe applied magnetic field at several different temperatures. For all the reconstituted ferritin samples a logarithmic function was fitted to the time dependence of the remanent magnetisation

$$
\sigma(t)=\sigma_{0}-S \ln \left(t-t^{\prime}\right)
$$


where $\sigma(t)$ is the specific magnetisation of the sample at time $t$, and the magnetic viscosity parameter, $S, \sigma_{0}$, and $t^{\prime}$ are variable parameters in the fitting process ${ }^{16}$. The mass specific magnetic viscosity parameter in zero field is plotted against temperature for all reconstituted ferritin samples in Figure 2. The characteristic blocking temperature, which is the temperature at which the greatest amount of moment is vulnerable to thermal activation (i.e. the peak in $S$ versus $T$ curve), clearly increases with iron loading. The mass-specific-magnetisation-weighted energy barrier distributions ${ }^{16}$ and the ironspecific-magnetisation-weighted energy barrier distributions ${ }^{16}$ of the reconstituted ferritins were determined from the time dependent behaviour of the magnetisation following methods described elsewhere ${ }^{16}$ and are shown in Figure 3 and Figure 4 respectively. The solid curves in both figures are fits of the equation

$$
\begin{aligned}
q(\Delta E) & =q_{1}(\Delta E)+q_{2}(\Delta E) \\
& =Q_{1} \exp \left(\frac{-\left(\ln (\Delta E)-\ln \left(\Delta E_{1}\right)\right)^{2}}{2[\Delta \ln (\Delta E)]^{2}}\right)+Q_{2} \exp \left(\frac{-\Delta E}{\Delta E_{2}}\right)
\end{aligned}
$$

to the data where $q(\Delta E)$ is the probability of a magnetic entity within the system having an energy barrier $\Delta E, q_{1}(\Delta E)$ and $q_{2}(\Delta E)$ are probabilities associated with two distinct contributions to the energy barrier distributions, and $\Delta E_{1}, \Delta E_{2}, Q_{1}$, and $Q_{2}$ are variable parameters used to fit the overall distribution to the data. The parameters derived from the fits are shown in Table II where the uncertainties on each of these parameters are those returned by the Levenberg-Marquardt algorithm used to fit the curve. These uncertainties are generally smaller than the true experimental errors. 


\section{Discussion}

TEM studies of the reconstituted ferritins indicate the presence of discrete electron dense particles with an average size that tends to decrease with decreased iron loading. In a previous study on these samples ${ }^{14}$ we found that the relationship between the mean particle volume and measured iron to protein ratio suggested a density of $6.4 \pm 0.6$ iron atoms per $\mathrm{nm}^{3}$ for the mineral particles. Taking the internal diameter of horse spleen apoferritin to be $8 \mathrm{~nm}$, these data imply a maximum possible iron loading per protein shell of $1700 \pm 200$ iron atoms. Native horse spleen ferritin is known to have average iron loadings of up to $3000 \mathrm{Fe}$ atoms per protein shell ${ }^{17}$ implying that the density of the iron oxyhydroxide mineral in the native protein is somewhat greater than that for the reconstituted mineral particles in this study. The higher density in the native protein may reflect the slower rate of iron uptake under physiological conditions.

Low temperature magnetic hysteresis measurements showed that the coercivity and field cooled hysteresis loop shift decrease rapidly with increasing temperature, with the loop shift vanishing near $10 \mathrm{~K}$. The onset of the loop shift has been associated in other nanoparticles ${ }^{8}$ with a "freezing" of disordered surface spins. Of note is the fact that the coercivity has a similar temperature dependence to the loop shift suggesting that the coupling with the frozen disordered surface spins makes core spin reversal more difficult. At temperatures greater than approximately $10 \mathrm{~K}$ the surface layer of spins may uncouple from the core spins within each ferrihydrite nanoparticle.

In previous experiments measuring the apparent magnetic energy barrier distribution in a sample of native horse spleen ferritin ${ }^{16}$ a distribution comprised of two components was observed and it was proposed that the log-normal component of the energy barrier 
distribution arises from the distribution of particle volumes and magnetic moments within the ensemble. Indeed, this finding is supported by the reconstituted ferritin results which show that the mode of the log-normal distribution $\left(\Delta E_{1}\right)$ exhibits a clearly increasing trend with increased iron loading (or particle size). The shifting of the peak of the lognormal component with particle size makes a numerical comparison of the relative areas of the exponential and log-normal components of the overall energy barrier distribution difficult. This difficulty is reflected in the extremely large errors associated with the fitting parameters for the smallest particle sizes shown in Table II.

The area under the experimentally measured iron-specific-magnetisation-weighted energy barrier distribution (Figure 4) from $8.97 \cdot 10^{-22} \mathrm{~J}$ upwards shows a decreasing trend with increasing iron loading and is consistent (agreement within 5\%) with the value of the initial thermoremanent iron specific magnetisation measured at $2 \mathrm{~K}$ for all the reconstituted ferritin samples. Thus, the smaller particles have a larger iron- specific magnetisation, suggesting that the larger particles have a better compensated antiferromagnetic spin-lattice. These results therefore support the concept of a higher concentration of uncompensated spins at the surface of the nanoparticles. The ferritin samples reconstituted at higher temperatures show slightly lower iron specific magnetisation in comparison to their counterparts synthesized at lower temperature, indicating a greater degree of spin compensation obtained in the more crystalline reconstituted ferritin samples. Furthermore, the magnetic-moment-weighted energy barrier distributions of the more crystalline samples appear to have a less significant lognormal component (see Figure 3 or Figure 4). If the magnetic entities at the surface of the mineral particle have a higher magnetic moment per Fe atom than the core entities (which 
have more complete compensation), the surface entities will contribute far more per Fe atom to the magnetic-moment-weighted energy barrier distribution. For the ferritin samples reconstituted at higher temperatures it is likely that the core entities have a greater degree of spin compensation than the ferritin samples reconstituted at lower temperatures because of their higher degree of structural order. Thus, the surface entities would be expected to contribute relatively more to the overall energy barrier distribution in the more crystalline samples, suggesting that the exponentially decreasing component of the energy barrier distribution may be associated with the surface of the particles.

\section{Conclusions}

In conclusion, ferrihydrite nanoparticles with different average particle size and degrees of crystallinity have been synthesised by controlling the iron loading per ferritin protein cage and the temperature of reconstitution respectively. The specific magnetisationweighted-energy barrier distribution in the reconstituted ferritin samples was measured and found to be comprised of two distinct distributions. Evidence that the log-normal component of this distribution arises from the distribution of particle volumes and moments within the ensemble is strongly supported by the increase of the mode of the energy barrier distribution with increasing particle size. The relatively larger contribution of the exponential distribution in the more crystalline reconstituted ferritin samples suggests that the exponential component of the energy barrier distribution may be associated with uncompensated spins at the surfaces of the particles. 


\section{Acknowledgements}

Funding was provided by the Australian Research Council (Grant No. DP0451535). 
TABLE I. The ferrihydrite particle size and iron content (in Fe atoms per ferritin shell) \pm one standard deviation in ferritins reconstituted with different iron loadings at different temperatures.

\begin{tabular}{ccccc}
\hline \hline $\begin{array}{c}\text { Nominal Fe atoms } \\
\text { per ferritin shell }\end{array}$ & \multicolumn{2}{c}{$\begin{array}{c}\text { Measured Fe atoms } \\
\text { per ferritin shell }\end{array}$} & \multicolumn{2}{c}{$\begin{array}{c}\text { Mean particle size } \\
(\mathrm{nm})\end{array}$} \\
\hline & $25^{\circ} \mathrm{C}$ & $50^{\circ} \mathrm{C}$ & $25^{\circ} \mathrm{C}$ & $50^{\circ} \mathrm{C}$ \\
100 & $19 \pm 0.6$ & $56 \pm 12$ & $3.40 \pm 0.74$ & $5.07 \pm 1.08$ \\
500 & $315 \pm 13$ & $396 \pm 12$ & $6.33 \pm 1.28$ & $6.03 \pm 1.30$ \\
1000 & $695 \pm 13$ & $688 \pm 12$ & $7.94 \pm 0.93$ & $6.03 \pm 1.30$ \\
1500 & $1100 \pm 120$ & $760 \pm 40$ & $8.74 \pm 0.96$ & $7.05 \pm 1.39$ \\
2000 & $1100 \pm 100$ & $\ldots$ & $7.54 \pm 1.08$ & $\ldots$ \\
\hline
\end{tabular}


TABLE II. Parameters derived from the fit of Equation 2 to the data shown in Figure 4 . The units for $Q_{1}$ and $Q_{2}$ are $10^{20} \mathrm{~T}^{-1}(\mathrm{~kg} \mathrm{Fe})^{-1}$ and for $\Delta E_{1}$ and $\Delta E_{2}$ are $10^{-21} \mathrm{~J}$. Also shown is the numerically calculated area under the curve (in $\mathrm{JT}^{-1}(\mathrm{~kg} \mathrm{Fe})^{-1}$ ) along with the percentage contribution to the total area from each of the components that make up the overall distribution.

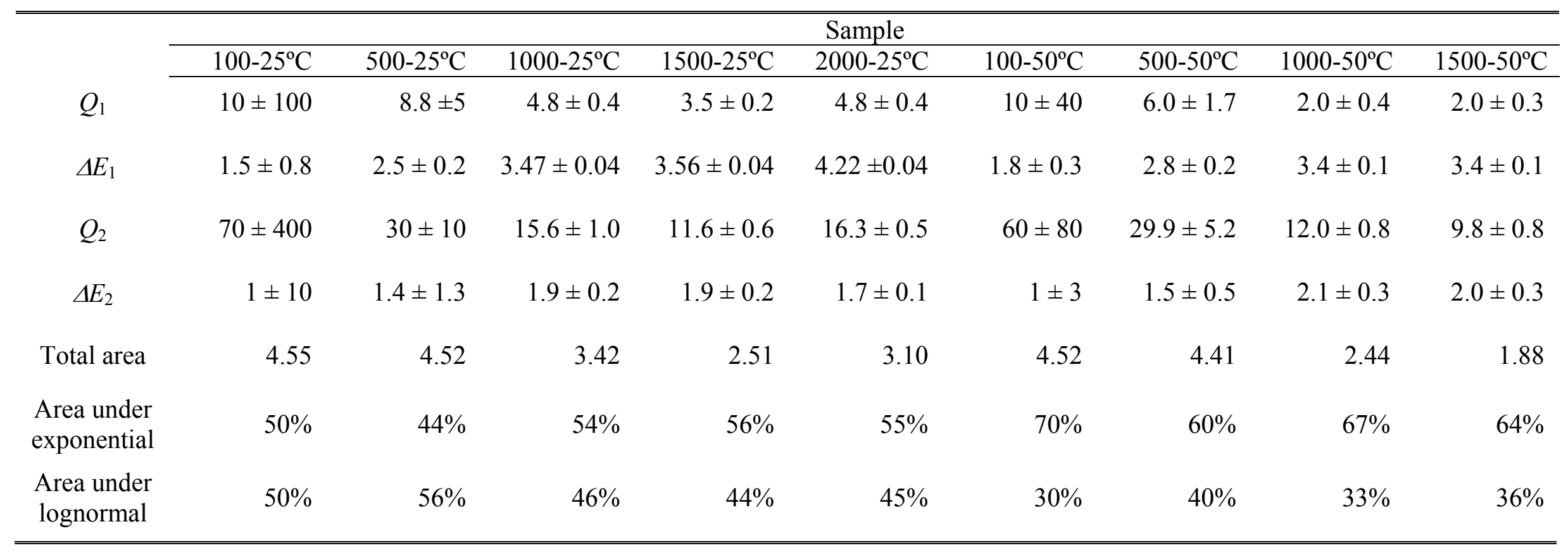




\section{Figure Captions}

\section{Figure 1}

Logarithmic time dependence of the iron specific magnetisation of two reconstituted ferritin samples (a) $1000-25^{\circ} \mathrm{C}$ and (b) $1000-50^{\circ} \mathrm{C}$. The solid circles are experimentally measured data points for the ferritins in zero field after removal of an applied field. The solid lines are fits of Equation 1 to the data.

\section{Figure 2}

Mass specific magnetic viscosity parameter, $S$, of the reconstituted ferritins (a) $25^{\circ} \mathrm{C}$ and (b) $50^{\circ} \mathrm{C}$ between temperatures of 2 and $22 \mathrm{~K}$.

\section{Figure 3}

Mass-specific-magnetisation-weighted energy barrier distributions for the (a) $25^{\circ} \mathrm{C}$ and (b) $50^{\circ} \mathrm{C}$ reconstituted ferritins. The experimentally measured data points are shown together with solid curves that are fits of Equation 2 to the data.

\section{Figure 4}

The iron-specific-magnetisation-weighted energy barrier distributions for (a) the $25^{\circ} \mathrm{C}$ and (b) the $50^{\circ} \mathrm{C}$ reconstituted ferritins. The experimentally measured data points are shown together with solid curves that are fits of Equation 2 to the data. 


\section{References}

3

S. Gider, D. D. Awschalom, T. Douglas, S. Mann, and M. Chaparala, Science 268, 77-80 (1995).

Mat. 226, 1315-1316 (2001). 
T. G. St. Pierre, P. Chan, K. R. Bauchspiess, J. Webb, S. Betteridge, S.

Walton, and D. P. E. Dickson, Coord. Chem. Rev. 151, 125-43 (1996).

W. Chua-anusorn, H.-R. Mun, J. Webb, N. T. Gorham, and T. G. St. Pierre, Hyp. Interact. 144/145, 279-288 (2002).

S. A. Makhlouf, F. T. Parker, and A. E. Berkowitz, Phys. Rev. B 55, 1471714720 (1997).

T. G. St. Pierre, N. T. Gorham, P. D. Allen, J. L. Costa-Kramer, and K. V. Rao, Phys. Rev. B 6502, 4436 (2002). (1986). 


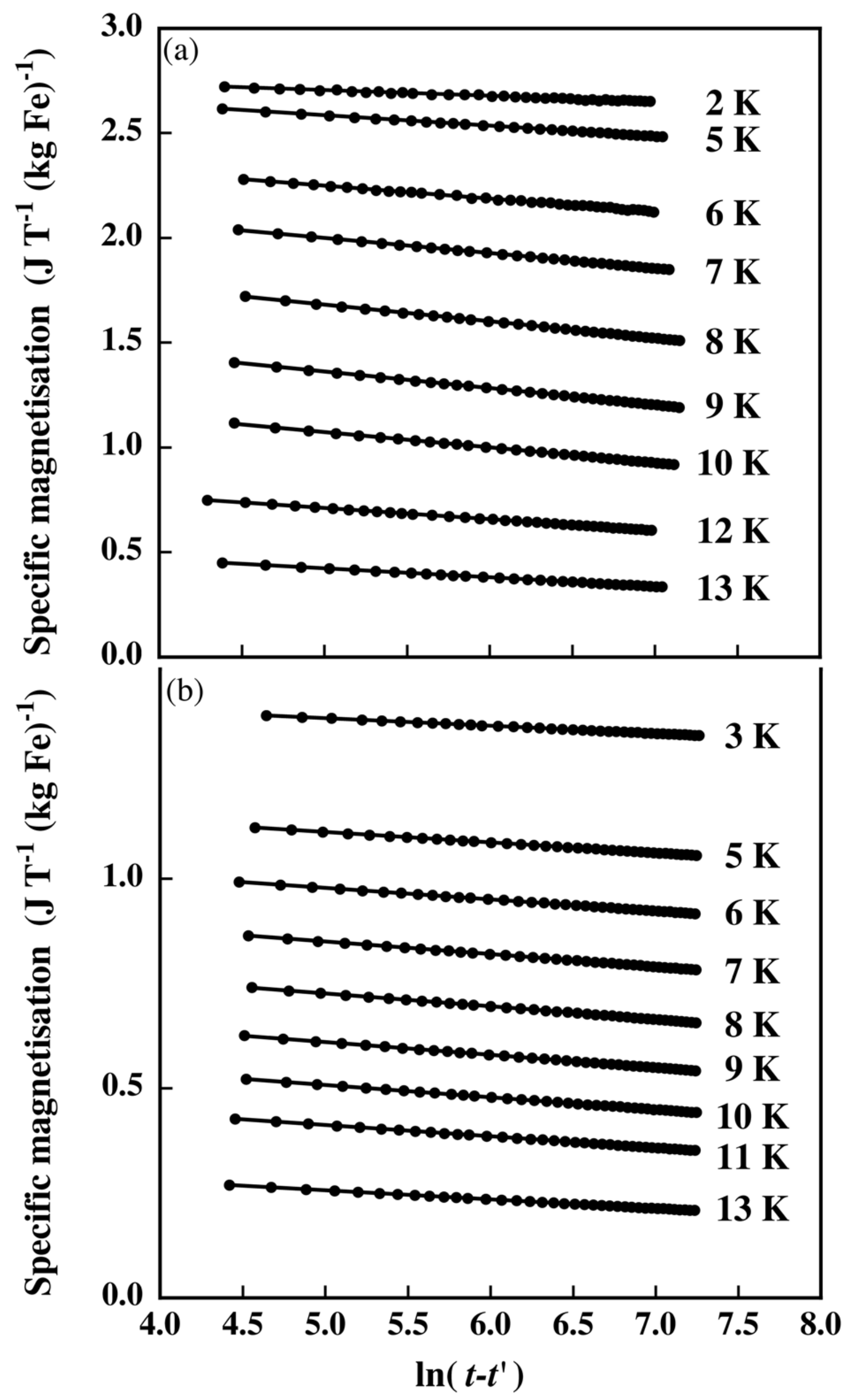




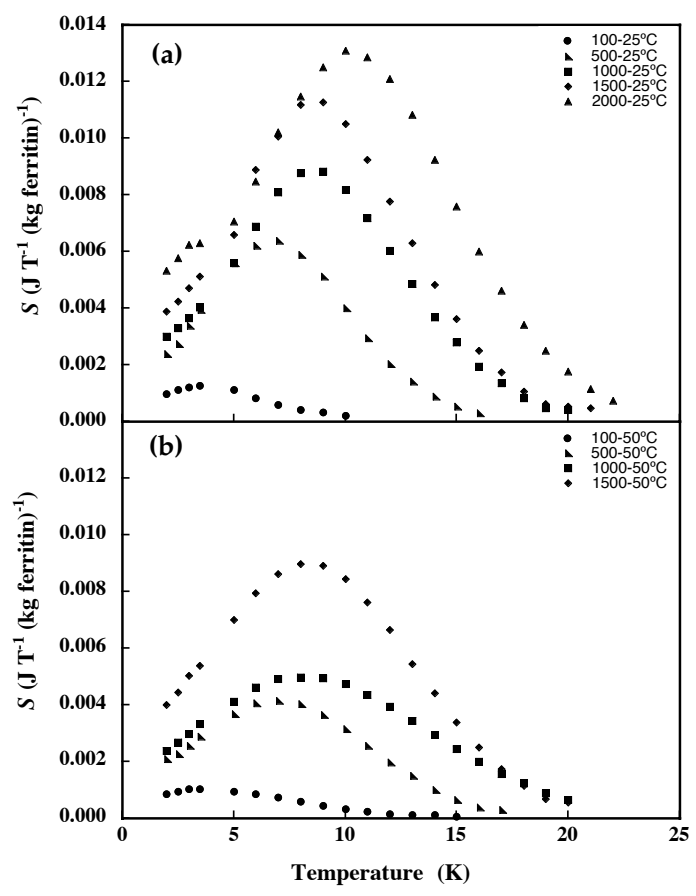

Figure 2 


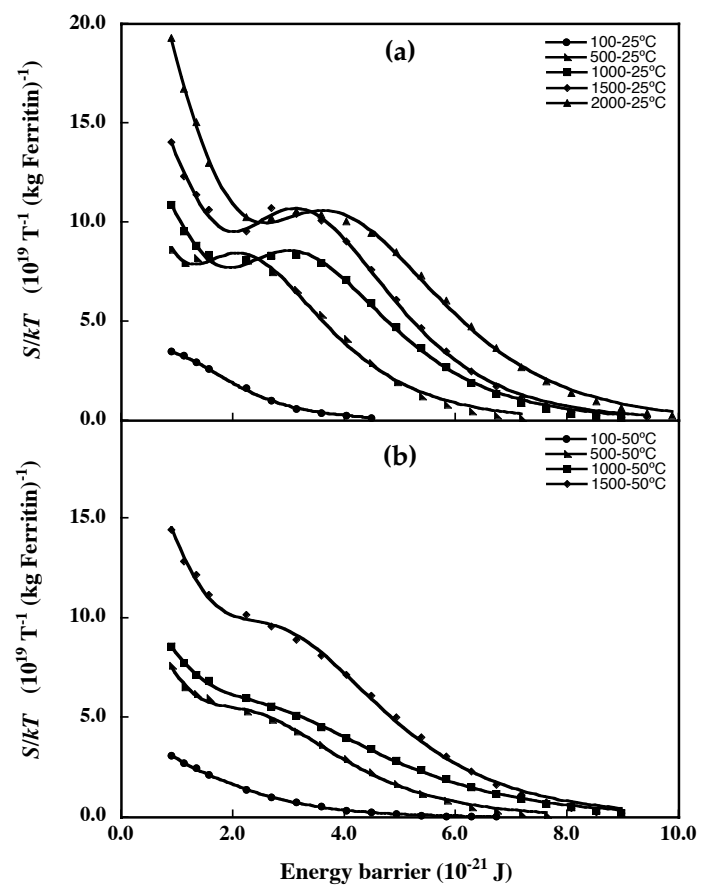

Figure 3 


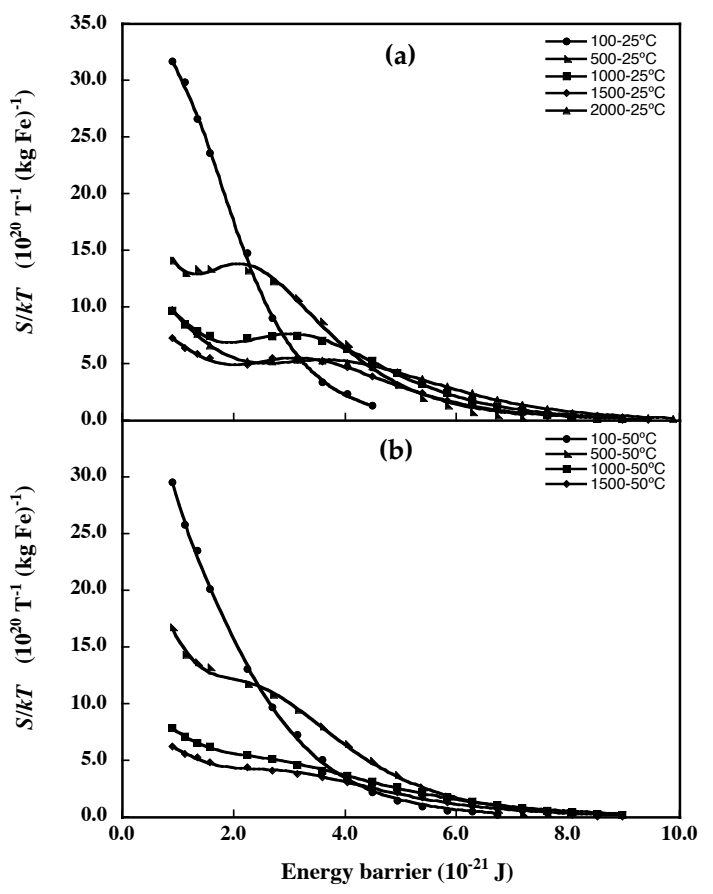

Figure 4 